Review

\title{
Trends in Flow-based Biosensing Systems for Pesticide Assessment
}

\author{
Beatriz Prieto-Simón ${ }^{1}$, Mònica Campàs ${ }^{1}$, Silvana Andreescu ${ }^{2}$ and Jean-Louis Marty ${ }^{1, *}$ \\ 1 BIOMEM group, Université de Perpignan, 52 Avenue Paul Alduy, 66860 Perpignan Cedex, France \\ 2 Department of Chemistry and Biomolecular Sciences, Clarkson University, Potsdam, NY 13699- \\ 5810, USA
}

Beatriz Prieto-Simón, e-mail: beatriz.prieto-simon@univ-perp.fr; Mònica Campàs, e-mail: campas@univ-perp.fr; Silvana Andreescu, e-mail: eandrees@clarkson.edu; Jean-Louis Marty, e-mail: jlmarty@univ-perp.fr

* Corresponding author: Tel: +33(0)468662254; Fax: +33(0)468662223

Received: 29 July 2006 / Accepted: 29 September 2006 / Published: 1 October 2006

\begin{abstract}
This review gives a survey on the state of the art of pesticide detection using flow-based biosensing systems for sample screening. Although immunosensor systems have been proposed as powerful pesticide monitoring tools, this review is mainly focused on enzyme-based biosensors, as they are the most commonly employed when using a flow system. Among the different detection methods able to be integrated into flow-injection analysis (FIA) systems, the electrochemical ones will be treated in more detail, due to their high sensitivity, simple sample pretreatment, easy operational procedures and real-time detection. During the last decade, new trends have been emerging in order to increase the enzyme stability, the sensitivity and selectivity of the measurements, and to lower the detection limits. These approaches are based on (i) the design of novel matrices for enzyme immobilisation, (ii) new manifold configurations of the FIA system, sometimes including miniaturisation or lab-on-chip protocols thanks to micromachining technology, (iii) the use of cholinesterase enzymes either from various commercial sources or genetically modified with the aim of being more sensitive, (iv) the incorporation of other highly specific enzymes, such as organophosphate hydrolase (OPH) or parathion hydrolase $(\mathrm{PH})$ and $(\mathrm{v})$ the combination of different electrochemical methods of detection. This article discusses these novel strategies and their advantages and limitations.
\end{abstract}

Keywords: enzyme inhibition, acetylcholinesterase (AChE), pesticide, flow-injection analysis (FIA) system 


\section{Introduction}

Among all the hazardous environmental compounds, pesticides are the most abundant in soil, water, the atmosphere and agricultural products. Due to their widespread presence, great environmental concerns have recently appeared around this type of pollution. In the past few decades, organophosphorus and carbamate insecticides, the two most commonly found pesticide groups, have been widely used in many crops due to their lower environmental persistence compared to organochlorines [1,2]. These effective broad-spectrum compounds used against insect and arthropod pests are highly toxic to humans by different routes of exposure, such as dermal absorption, ingestion or inhalation. Their efficiency as pesticides and their acute toxicity to humans and animals are based on their ability to inhibit a group of hydrolytic enzymes called esterases. Acetylcholinesterase (AChE) is essential for the central nervous system, being present in both humans and insects. This enzyme hydrolyses the acetylcholine neurotransmitter in the synaptic membrane in order to avoid its accumulation. AChE inhibition and the subsequent acetylcholine accumulation cause a marked dysfunction of many autonomic and behavioural systems, eventually leading to respiratory paralysis and death [3,4]. Furthermore, the continuous exposure to organophosphorus compounds has been linked to chronic fatigue syndrome [5]. Pesticides represent a real threat not only to living organisms but also to the environment, especially to ground and surface water. Their toxicity justifies the crucial need of accurate and reliable methods to monitor pesticide levels for safety considerations. Moreover, the area of biodefence is also interested in this field of research, since several organophosphate compounds can be used as nerve agents (i.e. sarin and soman gases) [6].

Due to the obvious health and environmental concerns, most countries have implemented strict legislations to control the use of pesticides in agricultural fields. Several organisations regulate the maximum levels of permissible pesticide residues in drinking water and in food for human and animal consumption. Among them, it is important to highlight the role of the Food and Agricultural Organization (FAO) of the United Nations, the World Health Organisation (WHO), the European Community (EU), the US Environmental Protection Agency (EPA) and the US National Institute for Occupational Safety and Health (NIOSH).

The FAO has developed a crop protection and government policy, published in the "International Code of Conduct on the Distribution and Use of Pesticides" [7]. The FAO together with the WHO have proposed maximum residue limits (MRLs) for pesticides in food and feed commodities moving in international trade [8]. Apart from providing these levels, they are also advising on organisation and management, publishing operational guidelines and manuals, and training personnel in the use of equipment and protocols for pesticide analysis, in order to stimulate fruitful discussions between national governments and interested organisations and promote the establishment of plant protection capabilities.

The EU regulates the marketing and use of plan protection products in water and in food. The EU Water Framework Directive aims for the assessment, monitoring and management of the water quality in rivers, lakes, groundwater and coastal beaches. This directive establishes that individual pesticides and their transformation products should be monitored at the $0.1 \mu \mathrm{g} / \mathrm{L}$ level and the total pesticide concentration cannot exceed $0.5 \mu \mathrm{g} / \mathrm{L}$ [9]. With regard to food, the Council Directives 76/895/EEC, 
86/362/EEC, 86/363/EEC and 90/642/EC and the Regulation (EC) No 396/2005 set the maximum levels of pesticides; their exceedance does not indicate a toxicological danger but an incorrect use [10].

The EPA recommends the use of new biopesticides or pesticides with low impact on the animal and human health and low risk for water contamination [11]. This organisation also reviews older pesticides in order to ensure that they meet current safety standards. Among the different laws, the "Food Quality Protection Act" (FQPA) of 1996 amended the "Federal Insecticide, Fungicide, and Rodenticide Act" (FIFRA) and the "Federal Food Drug, and Cosmetic Act (FFDCA)", which makes uniform requirements regarding processed and unprocessed foods with special emphasis on the protection of infants and children [12].

Finally, the NIOSH has included the acute occupational pesticide-related illness and injury into the "Sentinel Event Notification System for Occupational Risk" (SENSOR) program [13]. This program, also supported by the EPA, aims at building and maintaining occupational illness and injury surveillance capacity within state health departments. The derived "Pesticides National Aggregated Database" reports the number of acute occupational pesticide-related cases, performs in-depth investigations for case confirmation and develops preventive interventions aimed at particular industries or pesticide hazards.

Detection methods for monitoring the presence of these neurotoxic compounds require high sensitivity and accuracy, since pesticides are mostly found at trace levels. Although the traditional analytical techniques fulfil these requirements, they are not suitable for direct monitoring of pesticides in real samples. Gas chromatography, liquid chromatography, thin film chromatography and various spectroscopic techniques [14], coupled to selective detectors such as mass spectrometry, involve compound extraction, preconcentration and clean-up steps, which make them tedious, time-consuming, expensive and not suitable for in-field analysis. Moreover, highly trained technicians and specialised laboratories are required [15]. Another drawback is the fact that they do not measure the toxicological effect of the pesticides [16].

Bioanalytical assays, based on enzymatic or immunochemical principles, have been proposed as promising alternatives, as they are highly sensitive, selective, specific, rapid and reliable. Development of biological sensors based on these assays offer additional advantages such as the possibility to be incorporated in miniaturised and portable devices for in-field and/or on-line measurements, the low cost and the ease of operation even by non-skilled personnel [17,18]. Enzyme biosensors are based on inhibition measurements with cholinesterases or on direct measurements with organophosporate hydrolase $(\mathrm{OPH})$ or parathion hydrolase $(\mathrm{PH})$. Unlike enzyme sensors, immunosensors only detect the pesticide to which they are specific, instead of evaluating the total toxicity. Despite their clear advantages, the development of a successful biosensor has encountered several problems, such as low response stability (due to biomolecule leaking or deactivation), low mechanical stability, high diffusion resistance of the substrate/biocomponent assembly, interfering signals arising from other compounds present in real samples and, especially for cholinesterase-based biosensors, multi-stage procedures. Thus, the biosensor design is a key factor to overcome these drawbacks.

The combination of biosensors with flow-injection analysis (FIA) techniques offers the possibility to control the whole procedure, simplifying the sequence of steps and allowing an easier optimisation of the reaction conditions $[19,20]$. In the past 25 years, FIA has been the most widely proposed method 
of automation, due to its efficiency and versatility [21]. Compared to batch systems, flow-through based biosensing systems present the advantages of the reduction in analysis time, which allows a high sample throughput, and the possibility to work with small volumes of substrate and sample [22].

This article focuses on biosensors for pesticides coupled to FIA systems, with special emphasis on the enzyme-based strategies. Two approaches have been described for the integration of biosensor systems into a FIA configuration: i) with enzymatic reactors or enzyme-modified magnetic particles with a separate electrochemical detector, and ii) with biosensors integrated into the flow circuit. In both cases, the presence of immobilised enzyme considerably reduces the analysis cost. Moreover, the current achievements in microelectronics and micromechanics offer a valuable tool for the future miniaturisation of the whole system, this being the first step towards the development of portable field instrumentation.

\section{Flow-based immunosensing systems}

Immunoassay-based sensors, commonly named immunosensors, are an alternative to the chromatographic techniques. Immunoassays are based on antigen-antibody reversible affinity interactions and are characterised by the simplicity and the inherent sensitivity and specificity towards a particular pesticide. Other advantages are the rapidity and the relative low cost of the assays, the little sample pre-treatment and the possibility to analyse a large number of samples, which make them attractive for screening purposes. Their application to environmental monitoring is thus justified and has been extensively reviewed [23-25].

Flow-through immunosensors use the same principles as classical immunosensors. Apart from the automation, which confers precision and rapidity to the analysis, they also offer the possibility of regenerating the immunosurface, with the subsequent reagent saving. However, flow injection immunoanalysis (FIIA) has been scarcely developed and few articles are reported about this subject. Although most immunoassays use enzymes as labels, when incorporated into FIA systems fluorophores are more commonly found. It is necessary to distinguish between the configurations where the antibody or the antigen is immobilised onto the optical transducer (planar waveguide or optic fiber) [26] from those where the biomolecule is incorporated into an immunoreactor and the fluorescent signal arising from the affinity interaction is then analysed using an on-line optical detector [27-29]. Flow-based immunosensors have been applied to the analysis of several pesticides in real samples, such as chlorotriazines in river water [26], carbaryl in commercial drinking water and apple juice [27], and Irgarol 1051, an algaecide used as antifouling agent for boats, in bottle water, river water and seawater [29].

Although slightly beyond our scope, we consider it necessary to mention the electrochemical system developed by Bauer et al. [30]. They describe a bi-enzyme substrate-recycling biosensor incorporated into a FIA system for the zeptomole detection of alkaline phosphatase. The principle is based on the detection of the phenol produced by the enzymatic reaction and the subsequent signal amplification by the use of tyrosinase and glucose dehydrogenase. In order to demonstrate the applicability of this approach, they analyse the phenols produced in the dephosphorylation of the widely used herbicide 2,4-dichlorophenoxyacetic acid. However, they perform the immunoassay in a microtiter plate and not in the flow system. Despite this inconvenience, further development of the 
system by incorporating both the immunoassay and the electrochemical detector in the same system could be envisaged.

\section{Flow-based enzymatic sensing systems}

\subsection{Principle of the enzymatic-based sensor system}

Enzymatic detection of pesticides is most often based on cholinesterase inhibition. Although carbamate and organophosphate insecticides are the main cholinesterase inhibitors, this enzyme can also be inhibited by nicotine, fluoride, hypochlorite ions and heavy metals. Consequently, cholinesterase-based biosensors are not selective but give an indication of the global toxicity of a sample.

Carbamate and organophosphate compounds have some structural similarities with acetylcholine (ACh), the natural substrate of cholinesterase. The enzyme inhibition mechanism takes place in two steps: the enzyme-inhibitor complex reversible formation $\left(\mathrm{K}_{\mathrm{d}}=\mathrm{k}_{-1} / \mathrm{k}_{1}\right)$ and the carbamoylation or phosphorylation $\left(\mathrm{k}_{2}\right)$, which leaves the enzyme inactive [31]. The total mechanism is characterised by the inhibition rate constant $\mathrm{k}_{\mathrm{i}}=\mathrm{k}_{2} / \mathrm{K}_{\mathrm{d}}$ (Equation I):

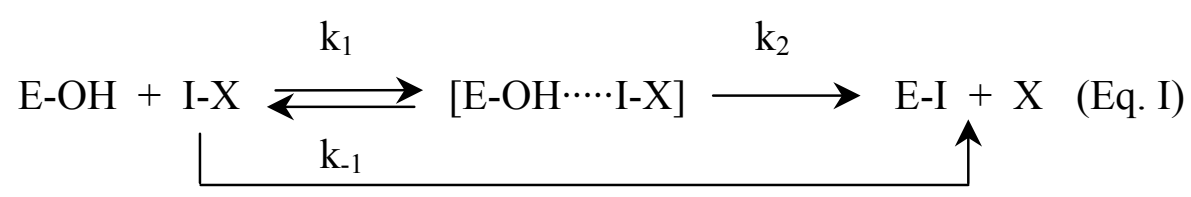

$\mathrm{k}_{\mathrm{i}}$

where $\mathrm{E}-\mathrm{OH}$ is the active enzyme, I-X the inhibitor, E-I the inactive enzyme and $\mathrm{X}$ the hydrolysable group of the inhibitor. The inhibiting mode of action of carbamate and organophosphate pesticides is different: whereas carbamates are reversible inhibitors, organophosphates are irreversible. The common protocol to measure the anti-cholinesterase activity of a pesticide is: 1) the measurement of the activity of the enzyme before inhibition $\left.\left(\mathrm{A}_{0}\right), 2\right)$ the incubation of the enzyme with the inhibitor for a period of time, and 3$)$ the measurement of the activity of the enzyme after inhibition $\left(\mathrm{A}_{\mathrm{I}}\right)$. The inhibition percentage is expressed as $I \%=\left(\mathrm{A}_{0}-\mathrm{A}_{\mathrm{I}}\right) \times 100 / \mathrm{A}_{0}$.

Acetylcholinesterase (AChE) hydrolyses the neurotransmitter ACh in the synaptic membrane, playing a fundamental role in nerve action in both human and insects. Butyrylcholinesterase (BChE) is another alternative, although less frequently used. $\mathrm{AChE}$ and $\mathrm{BChE}$ use $\mathrm{ACh}$ and butyrylcholine (BCh) as substrates, respectively, producing choline $(\mathrm{Ch})$ and the corresponding carboxylic acid (Equations II and III).

$$
\begin{aligned}
& \text { AChE } \\
& \mathrm{ACh}+\mathrm{H}_{2} \mathrm{O} \longrightarrow \mathrm{Ch}+\text { acetic acid } \quad \text { (Eq. II) } \\
& \text { BChE } \\
& \mathrm{BCh}+\mathrm{H}_{2} \mathrm{O} \longrightarrow \mathrm{Ch}+\text { butyric acid }
\end{aligned}
$$


Since $\mathrm{Ch}$ is not electrochemically active, the evaluation of the inhibition is based on the measurement of the $\mathrm{pH}$ change due to the acid formation. This $\mathrm{pH}$ change can be monitored with $\mathrm{pH}$ sensitive spectrophotometric indicators [32,33], pH-sensitive fluorescence indicators [34,35] or potentiometrically, with a $\mathrm{pH}$ glass electrode [36]. Other electrochemical strategies can also be used by combining the previous enzymes with choline oxidase $(\mathrm{ChO})$ and measuring the oxygen consumption with a Clark oxygen electrode [37] or the $\mathrm{H}_{2} \mathrm{O}_{2}$ production by amperometry.

In the development of amperometric biosensors, $\mathrm{ACh}$ and $\mathrm{BCh}$ are replaced by acetylthiocholine (ATCh) and butyrylthiocholine (BTCh), with the consequent thiocholine (TCh) production (Equations IV and $\mathrm{V})$.

$$
\begin{aligned}
& \mathrm{ATCh}+\mathrm{H}_{2} \mathrm{O} \stackrel{\mathrm{AChE}}{\longrightarrow} \mathrm{TCh}+\text { acetic acid (Eq. IV) } \\
& \mathrm{BTCh}+\mathrm{H}_{2} \mathrm{O} \stackrel{\mathrm{BChE}}{\longrightarrow} \mathrm{TCh}+\text { butyric acid (Eq. V) }
\end{aligned}
$$

$\mathrm{TCh}$ is electrochemically active and can be detected by anodic oxidation at $+0.6 \mathrm{~V} v \mathrm{~s} . \mathrm{Ag} / \mathrm{AgCl}$ (Equation VI) [38] (Figure 1).

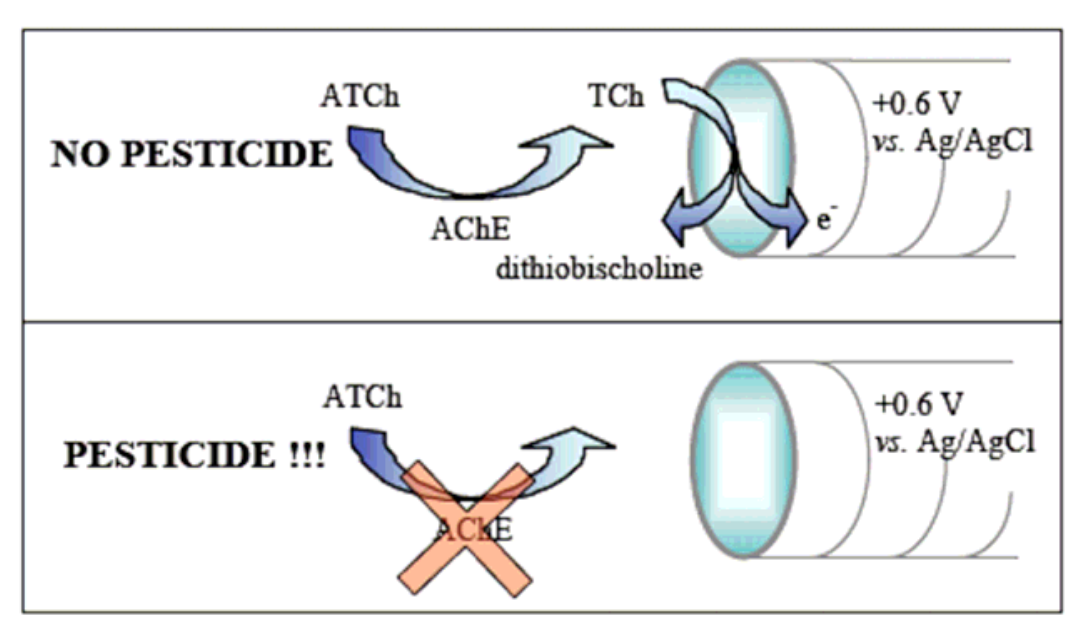

Figure 1. Scheme of the amperometric determination of pesticides based on AChE inhibition.

The use of electrochemical mediators, such as Meldola Blue (MB), hexacyanoferrate (III) or cobalt phthalocyanine (CoPC), allows for a lower working potential, minimising the contribution from other possible electroactive compounds of the sample [39-41].

anodic oxidation

$$
2 \mathrm{TCh}+\mathrm{H}_{2} \mathrm{O} \longrightarrow \text { dithiobischoline }+2 \mathrm{H}^{+}+2 \mathrm{e}^{-} \quad \text { (Eq. VI) }
$$


Another possibility in the development of amperometric biosensors is the use of $p$-aminophenyl acetate (PAPA) as AChE substrate, since the $p$-aminophenol (PAP) enzymatic product can be detected by anodic oxidation at $+0.25 \mathrm{~V} v$ s. $\mathrm{Ag} / \mathrm{AgCl}[42,43]$.

The enzyme source has a drastic effect on the biosensor performance; enzymes from different sources give different inhibition constants for the same pesticide. Generally, cholinesterases isolated from insects are more sensitive to insecticides than those extracted from from other sources. Additionally, the use of mutant enzymes with improved sensitivity and selectivity produced by genetic engineering techniques has contributed to a decrease in the limits of detection of the developed biosensors [41].

Enzyme regeneration is one of the key issues in the development of cholinesterase-based biosensors, since it solves the problem of multiple and/or continuous biosensor use. When the enzyme is reversibly inhibited by carbamate pesticides, regeneration can be achieved simply by incubation in fresh buffer. However, the irreversible inhibition produced by organophosphates implies the use of specific agents, such as pyridine-2-aldoxime (2-PAM), for enzyme reactivation [36,44].

Non-inhibition-based biosensors can also be developed for the detection of organophosphorous (OP) pesticides. In this case, organophosphate hydrolase $(\mathrm{OPH})$ is used. This enzyme catalyses the hydrolysis of a number of organophosphorous compounds, liberating protons (Equation VII) that can be detected potentiometrically [45]. When p-nitrophenol (PNP) is produced, amperometry can be used to detect its electrochemical oxidation $[46,47]$. Both detection systems can be combined with the aim of distinguishing the presence of organophosphorous in sample mixtures $[48,49]$.

$$
\mathrm{OP}+\mathrm{H}_{2} \mathrm{O} \stackrel{\mathrm{OPH}}{\longrightarrow} \text { hydrolysed } \mathrm{OP}+2 \mathrm{H}^{+}(+\mathrm{PNP})
$$

Compared to AChE- or BChE-based flow-through biosensors, the OPH-based ones do not provide a nanomolar LOD but, they have a faster response time and are very selective. Thus, they can be combined with AChE- (or BChE)-based biosensors in order to distinguish between organophosphorous and carbamate pesticides [37]. However, OPH is not commercially available, a fact that considerably limits its applicability.

The detection of parathion, an organophosphate pesticide, was also possible using parathion hydrolase (PH). This enzyme catalyses the hydrolysis of parathion to PNP, which has been detected by amperometry using potential steps $(+0.7 \mathrm{~V}$ for $2 \mathrm{~s}$ and $+0.85 \mathrm{~V}$ for $1 \mathrm{~s}, \mathrm{vs}$. $\mathrm{Ag} / \mathrm{AgCl})$ [50].

When biosensors are integrated into flow-through systems, covalent binding is preferred among the different existing enzyme immobilisation methods. Direct adsorption and entrapment into polymeric matrices provide uniform distribution of the enzyme molecules but the immobilisation is quite unstable and the enzyme tends to leach with time. On the contrary, covalent binding, although it may partially denature the enzyme, avoids to a great extent the enzyme leaching.

\subsection{Electrochemical-based systems}

Despite the fact that most of the work done on flow biosensor systems for the determination of pesticides has been based on amperometric detection, there are also some interesting studies that utilise 
potentiometric, conductimetric and combined detection techniques (potentiometric/conductimetric or potentimetric/amperometric), which are worth reviewing in detail. Furthermore, with the aim of enhancing the performance characteristics of the final systems, some authors have developed new electrochemical detection procedures, mainly based on modifications of the classical employed methods, obtaining results that fulfil their expectations. Table 1 shows the main characteristics of several studies that will be further discussed.

\subsubsection{Biosensor systems based on an electrochemical detection (single amperometric detection not included)}

\subsubsection{Single potentiometric-based systems}

Potentiometric enzyme-based biosensor systems have been recently reviewed [51,52]. Most of them are based on the determination of the $\mathrm{H}^{+}$liberated during the hydrolysis of acetylthiocholine by $\mathrm{AChE}$ using different $\mathrm{H}^{+}$-sensitive electrodes. Some of them are based on the immobilisation of the enzyme on controlled-pore glass (CPG)-based reactors $[53,54]$. Others use a single bead string reactor (SBSR), improving the mixing of the reagents with the sample and reducing the sample dispersion and the flow resistance $[55,56]$. It was demonstrated that the oxidation of the organophosphorous compounds to their oxon forms by bromine, prior to analysis, enhances the sensitivity of the $\mathrm{pH}$ measurement and decreases the detection limit [36].

Ivnitskii et al. [57] used a solid-state AChE sensor instead of a pH-sensitive electrode. Solid-state electrodes enable analyses at high ionic strength and high buffer capacity, but they need an electrochemical electron mediator, due to the limited electronic transfer between the enzymatic membrane and the electrode surface. The authors incorporated hexacyanoferrate (III) in the working solution and measured the potential change inside the membrane due to the reaction between the enzyme and the mediator. Furthermore, the presence of the mediator overcomes other problems, such as sensitivity to interferences and slight changes of ionic strength and buffer capacity.

A recent work that requires special attention is that carried out by Nikolelis et al. [58], where the enzyme AChE was incorporated inside air stable lipid films. A novel detection technique was also used. In this case, the $\mathrm{pH}$ change, responsible for dynamic alterations of the electrostatic fields and phase structure of the bilayer lipid membranes, produces transient ion currents, which are measured with a glass electrode. High sensitivity and low detection limits were achieved working with a stopped-flow injection analysis mode, as it extends the contact time between inhibitor and enzyme.

Other potentiometric studies are based on the use of OPH enzyme. For example, Rainina et al. [45] entrapped recombinant E. coli onto gel spheres retained inside a reactor by using Poly(Vinyl) Alcohol (PVA) and cryoimmobilisation. The use of cells to express $\mathrm{OPH}$, favours the stability of the enzyme providing it with physical protection, while the cryoimmobilisation technique creates macropores that allow an adequate permeability for substrates and reaction products. Moreover, the cells can be used repeatedly due to their high mechanical strength. An additional advantage is the non-solubility of the gel matrix in organic solvents, which in principle allows the monitoring of reactions in a wide range of solvents. 


\begin{tabular}{|c|c|c|c|c|c|}
\hline 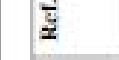 & $\$$ & $\approx$ & $\because$ & $\% 8$ & a \\
\hline$\frac{\sqrt{2}}{\frac{2}{2}}$ & 1 & 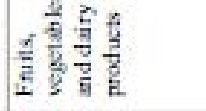 & 1 & 1 & $\frac{\pi}{3}$ \\
\hline$\frac{2}{3}$ & i & 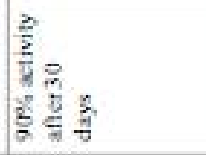 & 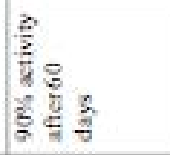 & 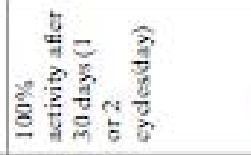 & 1 \\
\hline$\hat{\mathrm{t}}$ & 1 & 1 & 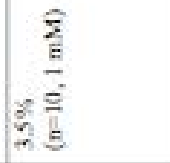 & 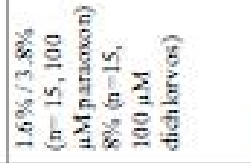 & व. \\
\hline 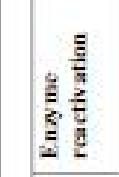 & 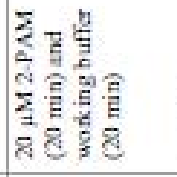 & 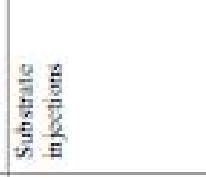 & $\frac{\frac{\pi}{8}}{\frac{8}{8}}$ & $\frac{\frac{8}{8}}{\frac{2}{2}}$ & i \\
\hline 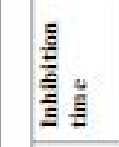 & 量 & E & $\frac{\frac{\pi}{g}}{\frac{g}{2}}$ & $\frac{\bar{g}}{\mathrm{~g}}$ & 量罳 \\
\hline ఏิ & 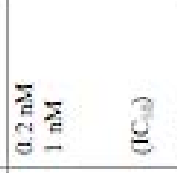 & 를 & 1 & 产 & 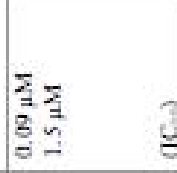 \\
\hline 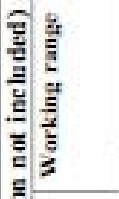 & 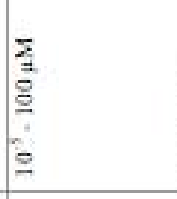 & $\frac{\bar{z}}{\overline{3}}$ & $\frac{2}{\frac{1}{8}}$ & 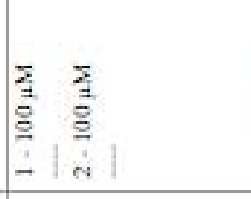 & 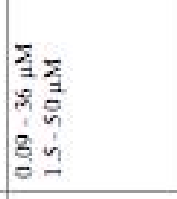 \\
\hline 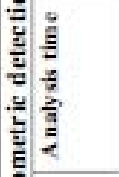 & 1 & 1 & 吾 & $\frac{\text { 量 }}{v}$ & 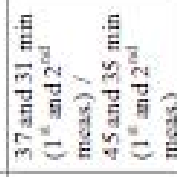 \\
\hline 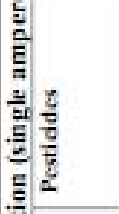 & 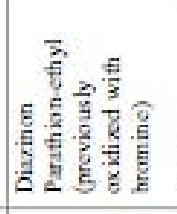 & 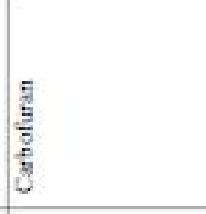 & $\begin{array}{l}\frac{\pi}{2} \\
\frac{8}{2} \\
2\end{array}$ & 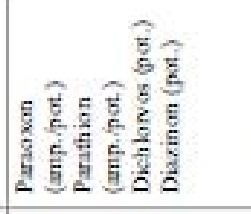 & 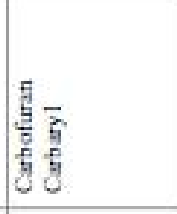 \\
\hline 宸 & 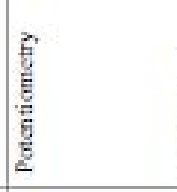 & 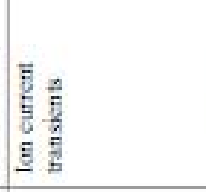 & है & 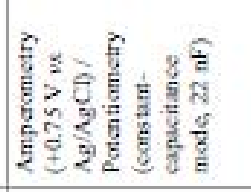 & 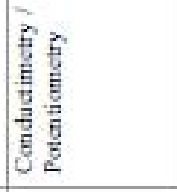 \\
\hline 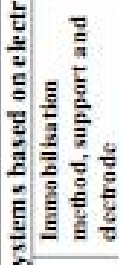 & 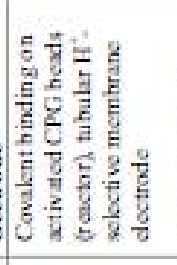 & 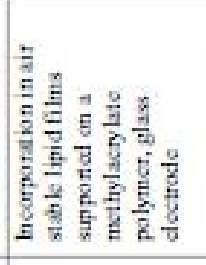 & 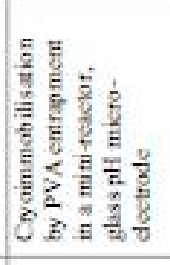 & 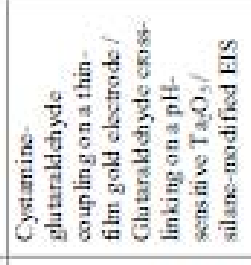 & 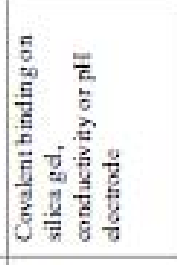 \\
\hline 这 & 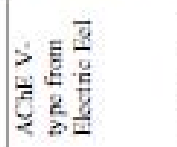 & 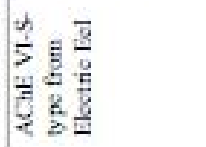 & 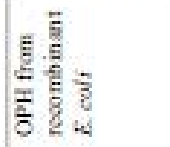 & 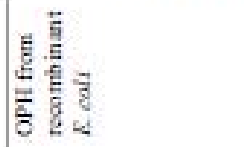 & 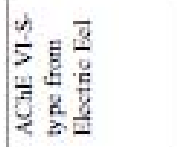 \\
\hline
\end{tabular}




\begin{tabular}{|c|c|c|c|c|c|c|c|c|c|}
\hline$\vec{\Xi}$ & 8 & $\Rightarrow$ & $\approx$ & z & 8 & 2 & 8 & $\vec{\nabla}$ & 8 \\
\hline 送 & 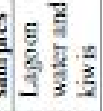 & & 1 & 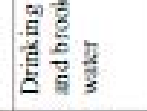 & 1 & 1 & $\frac{5}{3}$ & $\frac{\pi}{3}$ & 1 \\
\hline 竞 & 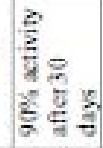 & & 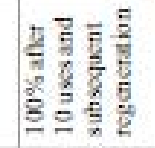 & 1 & 1 & 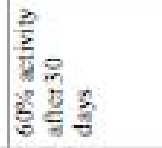 & I & 1 & 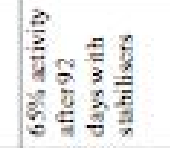 \\
\hline की & 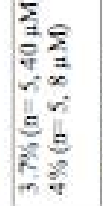 & 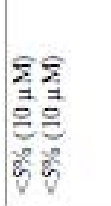 & 1 & 1 & 1 & 1 & 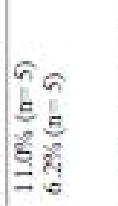 & 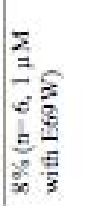 & 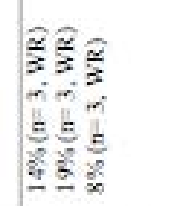 \\
\hline 政 & 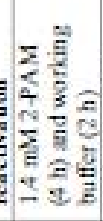 & 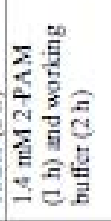 & 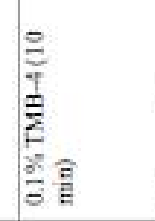 & 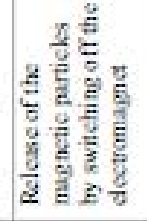 & 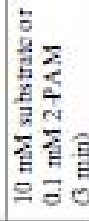 & 11 & 1 & 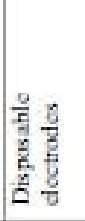 & 1 \\
\hline 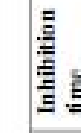 & & 1 & $\underset{E}{\underline{E}}$ & 恶 & 悬 & 产 & 量 & 至 & है \\
\hline อิ & 望至 & 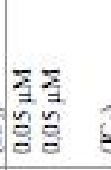 & 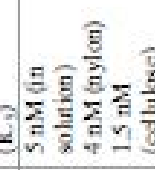 & 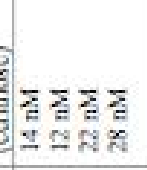 & 章 & क & 츨 & 疍 & 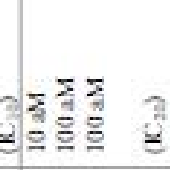 \\
\hline 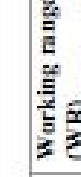 & 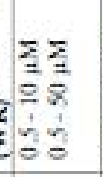 & 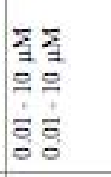 & 1 & 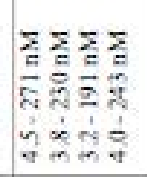 & $\begin{array}{l}\overline{1} \\
3 \\
8 \\
0 \\
0 \\
0\end{array}$ & 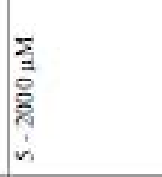 & 1 & $\frac{\pi}{3}$ & $\begin{array}{l}\Sigma \Sigma 2 \\
005 \\
1 \\
005\end{array}$ \\
\hline 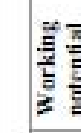 & $\begin{array}{l}3 \\
3 \\
3 \\
3 \\
9\end{array}$ & 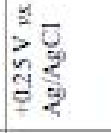 & 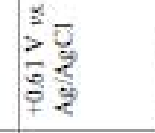 & 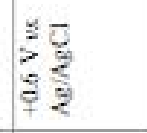 & $\begin{array}{l}50 \\
30 \\
30 \\
7=2\end{array}$ & 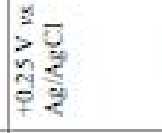 & $\begin{array}{l}5 \\
02 \\
02\end{array}$ & 1 & $\begin{array}{l}5 \overline{3} \\
>3 \\
y \\
72\end{array}$ \\
\hline 吾 & $\begin{array}{l}8 \\
\frac{8}{3} \\
\text { d }\end{array}$ & 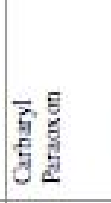 & $\frac{\text { E }}{\frac{5}{5}}$ & 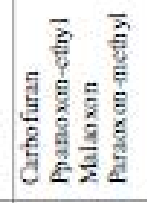 & $\frac{n}{6}$ & 旅 & ह & 韵 & 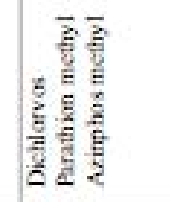 \\
\hline 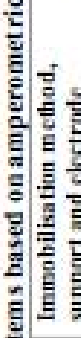 & 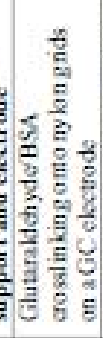 & 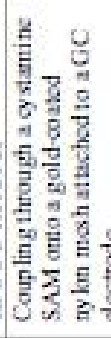 & 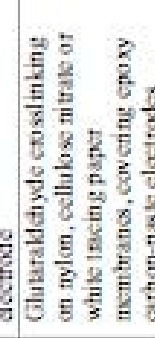 & 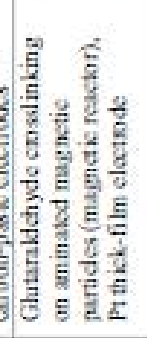 & 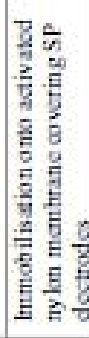 & 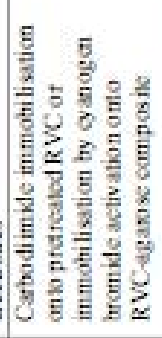 & 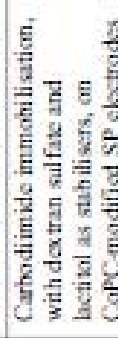 & 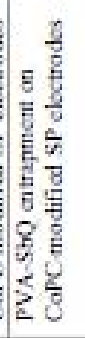 & 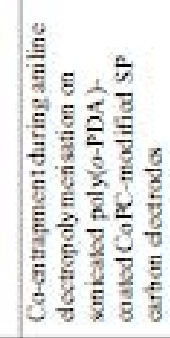 \\
\hline 递 & 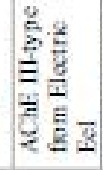 & 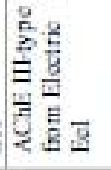 & 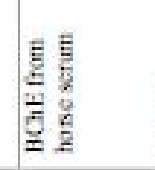 & 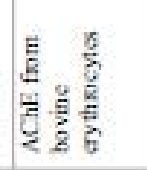 & 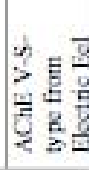 & 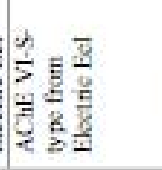 & 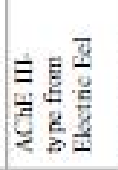 & 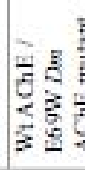 & 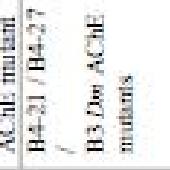 \\
\hline
\end{tabular}




\begin{tabular}{|c|c|c|c|c|c|c|c|c|c|}
\hline$\varepsilon$ & $\approx$ & R & $\vec{F}$ & 3 & $\%$ & $F$ & $\$$ & $f_{7}$ & 8 \\
\hline 1 & 1 & 1 & 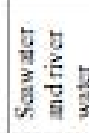 & 1 & $\frac{y}{2}$ & 1 & 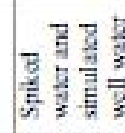 & & 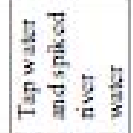 \\
\hline 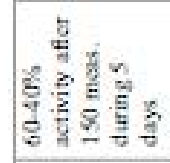 & 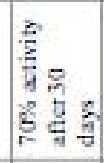 & 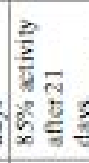 & 然 & 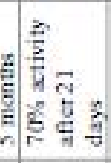 & 1 & I & 1 & 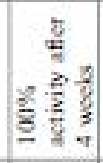 & 1 \\
\hline 28 & 1 & 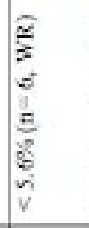 & 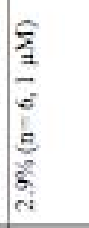 & 1 & 1 & 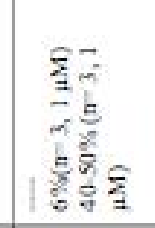 & 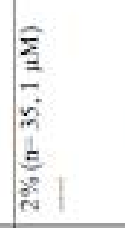 & 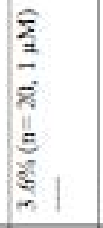 & 1 \\
\hline 1 & 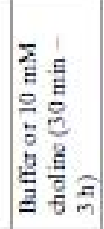 & 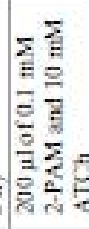 & 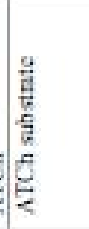 & 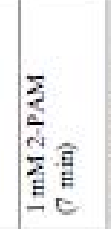 & 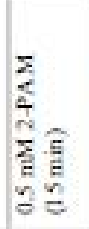 & 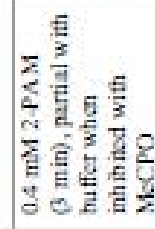 & $\frac{7}{8}$ & $\frac{7}{\frac{\pi}{8}}$ & $\frac{7}{\frac{3}{2}}$ \\
\hline 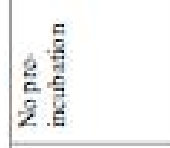 & 童 & E & $\begin{array}{l}\frac{E}{E} \\
\underline{E} \\
\end{array}$ & 1 & 1 & 偪量 & $\frac{3}{8}$ & $\begin{array}{l}\frac{8}{8} \\
\frac{8}{8} \\
\frac{8}{2}\end{array}$ & $\begin{array}{l}\frac{3}{3} \\
\frac{2}{2} \\
\frac{3}{2} \\
\end{array}$ \\
\hline 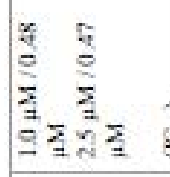 & 츨 & ${ }_{2}$ & $\stackrel{\bar{E}}{\stackrel{z}{E}}$ & 店 & $\bar{I}$ & 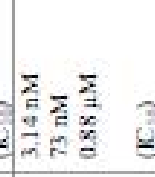 & 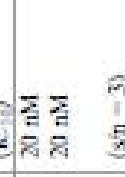 & $\frac{5}{3}$ & $\begin{array}{l}3 \\
\vdots \\
0\end{array}$ \\
\hline 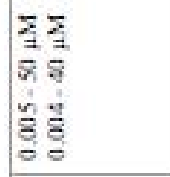 & \begin{tabular}{l}
2 \\
$\vdots$ \\
\hdashline \\
$\vdots$ \\
$\vdots$
\end{tabular} & 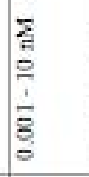 & $\begin{array}{l}\frac{\pi}{3} \\
\frac{\alpha}{2} \\
\frac{1}{0}\end{array}$ & 1 & $\begin{array}{l}\Sigma z \\
0 \% \\
0 \% \\
00\end{array}$ & 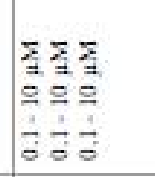 & 1 & 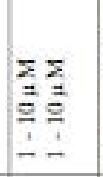 & $\begin{array}{l}2 \\
3 \\
1 \\
0 \\
3 \\
0 \\
0\end{array}$ \\
\hline 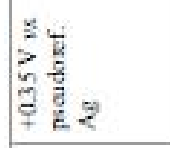 & 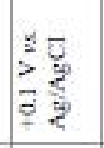 & 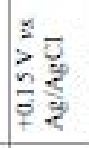 & 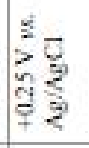 & 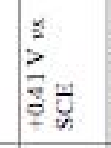 & 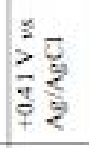 & 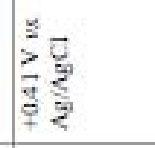 & $\begin{array}{l}5 \\
5 \\
3 \\
0 \\
7\end{array}$ & $\begin{array}{l}1 \\
20 \\
32 \\
02 \\
72\end{array}$ & 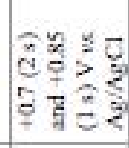 \\
\hline 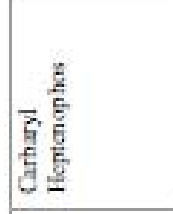 & 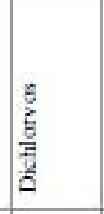 & 2 & 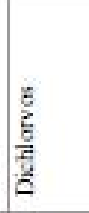 & E & 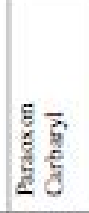 & 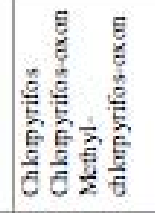 & 量 & 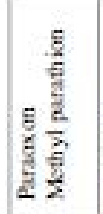 & 镸 \\
\hline 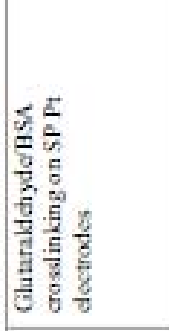 & 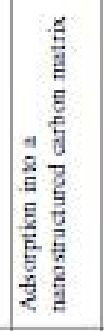 & 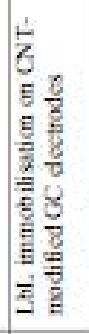 & 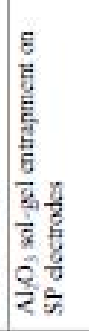 & 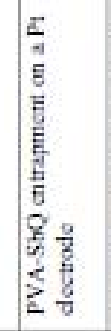 & 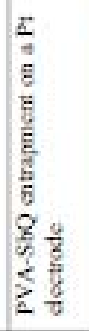 & 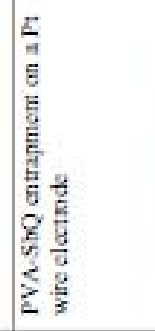 & 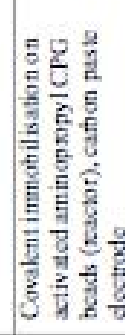 & 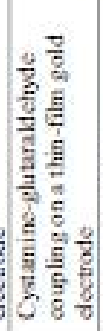 & 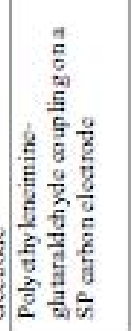 \\
\hline 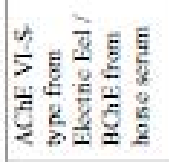 & 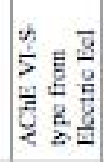 & 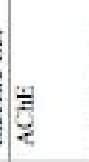 & $\begin{array}{l}\frac{2}{5} \\
\frac{2}{2} \\
\sum\end{array}$ & 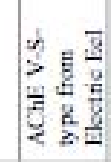 & & 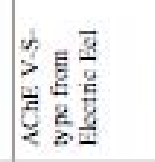 & 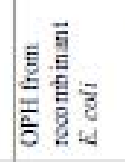 & 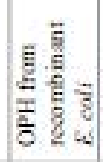 & है \\
\hline
\end{tabular}




\subsubsection{Dual potentiometric/amperometric-based systems}

In a more recent publication, the same authors [37] combined a potentiometric and an amperometric detector in order to discriminate between organophosporous and carbamate pesticides. Three enzyme reactors were used with $\mathrm{AChE}, \mathrm{ChO}$ and $\mathrm{OPH}$ immobilised on activated silica gel. First, the OPHbased reactor hydrolysed only organophosphorous pesticides. Then, the subsequent AChE/ChO-based system hydrolysed the remaining pesticides in the solution. The potentiometric measurement gave the concentration of organophosphorous pesticides, while the amperometric measurement indicated the concentration of carbamate pesticides. Although this approach allowed the discrimination between both types of neurotoxins, it was not possible to quantify the concentrations of each pesticide. Hence, the biosensor has to be used as a tool to screen the toxicity of the sample, prior to the analysis with a conventional chromatographic technique.

Similarly, a dual potentiometric/amperometric biosensor system was proposed [48,49], based on (1) the potentiometric detection of protons liberated by organophosphorous pesticides upon OPH hydrolysis and (2) the amperometric detection of $p$-nitrophenol produced by some pesticides, such as paraoxon and parathion. The combination of both transducers makes it possible to distinguish some organophosphorous pesticides in sample mixtures. It is important to notice that short response times (less than 1 minute) were obtained, even though no mutual influence of the sensor signals was observed, opening the possibility to design powerful cross-reactive biosensor arrays, the discrimination was still not high enough to give more details than a screening assay.

\subsubsection{Dual potentiometric/conductimetric-based systems}

The work done by Suwana-ard et al. [59] was based on the combination of a potentiometric and a conductimetric detector. Taking advantage of the best performance characteristics of each detection system, i.e. the high inhibition percentage, the low RSD for the potentiometric detection and the short analysis time for the conductimetric detection, carbofuran and carbaryl were detected in water samples without any sample preconcentration, with good sensitivity and low detection limits. But, like before, individual concentrations can not be determined and the biosensor system is only useful as a screening tool.

\subsubsection{Single conductimetric-based systems}

Conductimetry is usually based on the measurement of the number of ions after the hydrolysis of acetylcholine by AChE [59]. A more sophisticated system, applied to the paraoxon determination, is based on the conversion of acetate (coming from the acetylcholine hydrolysis) in acetic acid (when mixed with sulphuric acid), its passage as a gas through a diffusion membrane, and the subsequent conductimetric detection [60]. The main advantage of this system was the high selectivity and the high throughput (10 samples/h) due to the short incubation periods. Moreover, the total enzymatic regeneration was achieved using a solution of 1,1'-trimethylenebis[4-(hydroxyiminomethyl) pyridinium bromide] (TMB-4).

\subsubsection{Single amperometric-based systems}


Despite the complications of the amperometric systems, their better sensitivity has led to the development of a higher number of these devices compared to the potentiometric ones. These have been used in batch as well as in flow conditions. Most of the work has been directed to improve the stability of the enzyme, while increasing the sensitivity and lowering the detection limits. An example to improve the enzyme stability was based on the use of plant tissues enriched with AChE, which provide an optimum environment for the enzyme, also reducing the preparation costs [61].

One of the main problems encountered in amperometric-based sensors is the high working potential required to detect thiocholine. This high overpotential represents an important drawback, as other species present in the sample will be also oxidised, strongly affecting the selectivity. Moreover, some oxidation byproducts can adsorb onto the electrode surface, causing electrode fouling and decreasing the measured current.

\subsubsection{Use of alternative enzyme substrates}

Some authors have used alternative substrates for AChE, such as 4-aminophenyl acetate (PAPA). In this system, the resulting 4-aminophenol product can be easily oxidised at low potentials $(+0.25 \mathrm{~V} v \mathrm{~s}$. $\mathrm{Ag} / \mathrm{AgCl})[42,62,63]$. The use of a pre-incubation step between enzyme and inhibitor in the absence of substrate also improved the selectivity, as the possible electroactive species present in the sample (such as uric and ascorbic acid and benzaldehyde) were rinsed away with the carrier stream prior to the analysis. Natural water and kiwi fruit samples were spiked with carbaryl, and the results obtained with the biosensor were validated with UV determination, showing a good agreement. One year later, the same authors improved the enzyme immobilisation support, covering the nylon mesh with a thin layer of gold [43]. This new support, easy to manipulate due to its high tensile strength and flexibility, increased the enzyme stability without compromising the substrate permeability. Thus, lower flow rates could be used, which increased the enzyme/inhibitor contact time and hence the sensitivity. In addition, the rapid solute diffusion did not affect peak width, allowing a reasonable sample throughput.

\subsubsection{Use of membranes}

Evtugyn et al. [38] proposed the use of replaceable membranes to avoid electrode fouling. In this work, BChE was immobilised onto three different supports: nylon, cellulose nitrate and white tracing paper. Results were compared with the system containing BChE in solution. As it can be seen from Figure 2, higher sensitivity and lower detection limits were achieved when working with immobilised enzyme. This was explained by the sorptional preconcentration of the hydrophobic diazinon on the membrane surface while it is flowing through the cell. Thus, the performance characteristics of each biosensor depended on the hydrophobicity of the membrane material. It seems evident that the applied working potential $(+0.61 \mathrm{~V}$ vs. $\mathrm{Ag} / \mathrm{AgCl})$ would passivate the electrode surface making necessary an electrochemical cleaning treatment. Nevertheless, the use of epoxy-carbon electrodes covered with the mentioned enzymatic membranes eliminated this problem, as the residual current due to the iodide oxidation (counter ion of the substrate), which is oxidised at the same potential as thiocholine, became negligible. The explanation to these results is that the membrane has a different permeability toward various electroactive species: whereas iodide must diffuse to the electrode surface from the flowing 
carrier through the membrane, thiocholine is the product of the enzymatic reaction that takes place inside the membrane. The oxidation of both species can thus be discriminated choosing the appropriate working potential. Although the membranes were described as replaceable, $90 \%$ of enzymatic regeneration was achieved after 10 min of incubation using TMB-4, allowing the use of the same membrane for at least 20 determinations.

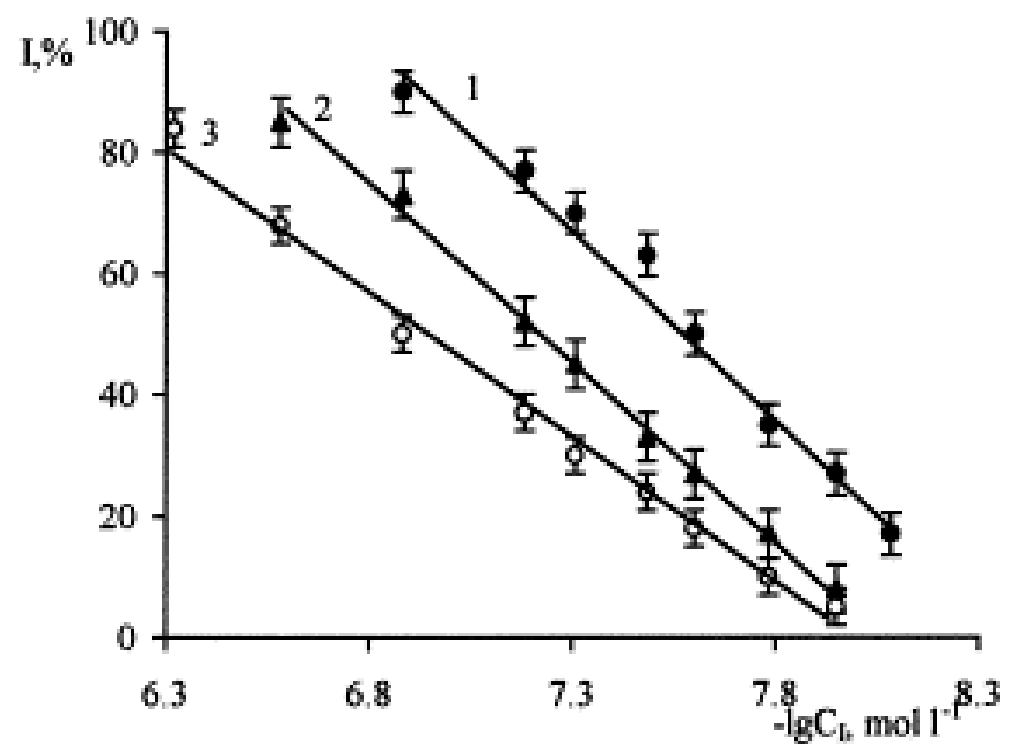

Figure 2. Calibration curves of diazinon determination after 10 min incubation: (1) cellulose nitrate; (2) nylon membrane; (3) enzyme solution. Reprinted from [38] with permission from Elsevier.

\subsubsection{Study of the counter ion effect}

The effect of the counter ion of the substrate was studied by Günter et al. [64]. They compared the effect of acetylthiocholine iodide (ATChI) and chloride (ATChCl) on the electrochemical response. When ATChI was used, a strong increase of the blank signal was noticed at $+0.2 \mathrm{~V}$. Due to these interferences, $\mathrm{ATChCl}$ was chosen as the best substrate. Only a few interferences were detected at the working potential $(+0.6 \mathrm{~V})$ when determining pesticides in drinking and brook waters, thus showing a good correlation with the colorimetric measurement. However, fouling effects caused by dimerised products coming from the thiocholine oxidation were not avoided.

\subsubsection{Use of electron mediators}

The easiest way to solve the overpotential problem seems to be the use of an electron mediator, which facilitates the electron transfer. In this case, the working potential of the enzyme electrode is determined by the oxidation potential of the mediator. Most of the studies with mediators are based on the use of screen-printed electrodes (SPEs), which are mass-produced at low cost, favouring their disposability. Neufeld et al. [40] developed a disposable micro-flow injection electrochemical biosensor, based on an AChE-modified nylon membrane attached to a SPE with hexacyanoferrate (III) in solution. The presence of this mediator allowed the determination of dichlorvos at $+0.3 \mathrm{~V} v s$. 
$\mathrm{Ag} / \mathrm{AgCl}$. Since the electrode was continuously washed by the flow stream, it was not necessary to clean it between repetitive measurements. This simplified the procedure and reduced the analysis time. Even though after inhibition, biosensor regeneration was not completely achieved, this did not represent a limitation, since the biosensors were designed as disposable.

Khayyami et al. used Meldola Blue (MB) as electron mediator [39]. Although MB allows working at $-0.1 \mathrm{~V}$ vs. $\mathrm{Ag} / \mathrm{AgCl}$, they applied a working potential of $+0.25 \mathrm{~V}$ in order to accelerate the oxidation rate. At this potential, background interference was higher, but the utility of the mediator was proved, since unwanted contributions to the signal from other electroactive compounds were minimised. On the other hand, the novelty of this work was the introduction of a new electrode material for enzyme immobilisation. Although simple reticulated vitreous carbon (RVC) was tested, best results were achieved when RVC was combined with superporous agarose. The final material showed a high enzyme binding capacity, a low background current, the possibility to work under appropriate flow conditions and the possibility to reuse the biosensors. On the other hand, the main disadvantage of this material was the slow thiocholine, $\mathrm{MB}$ and pesticide diffusion within the agarose gel, which made the response peaks slightly wider. Another inconvenience is the shorter contact time between enzyme and inhibitor, which compromises sensitivity in pesticide detection. Nevertheless, the authors proposed to manipulate the flow characteristics as a way to lower the limits of detection.

Rippeth et al. [65] avoided the problems related to the electrode fouling by using cobalt phthalocyanine (CoPC)-modified SPEs, which allowed working at $0 \mathrm{~V} v \mathrm{~s}$. $\mathrm{Ag} / \mathrm{AgCl}$, thus improving the selectivity. Furthermore, the replacement of the electrode after each measurement eliminated the need for enzyme regeneration. In order to improve the stability of the enzyme, the authors used polyelectrolytes jointly with polyhydroxyl compounds, holding the tertiary structure of the protein by electrostatic bonds. Best results were obtained when combining negatively-charged dextran sulphate with lactitol. The method was validated using river water samples and GC/MS detection. Although the method can be used for pesticide screening, it is necessary to know which pesticide is present in the sample, since each pesticide generated a different calibration curve. Another work using CoPCmodified SPEs was done by Bucur et al. [41]. The authors used AChE extracted from different sources as well as genetically modified mutants. The method is based on the combined effect of two enzymes, which enhances its selectivity: a highly sensitive Drosophila melanogaster mutant, which is inhibited by omethoate and by interferences, and a commercial AChE from Electric Eel, which is equally affected by interferences but is resistant to pesticides. Figure 3 clearly shows the different sensitivity of wild-type and mutant Drosophila melanogaster AChEs. This approach opens the way of designing devices able to discriminate among insecticides and all other interfering compounds by combining information obtained from different AChE enzymes. 


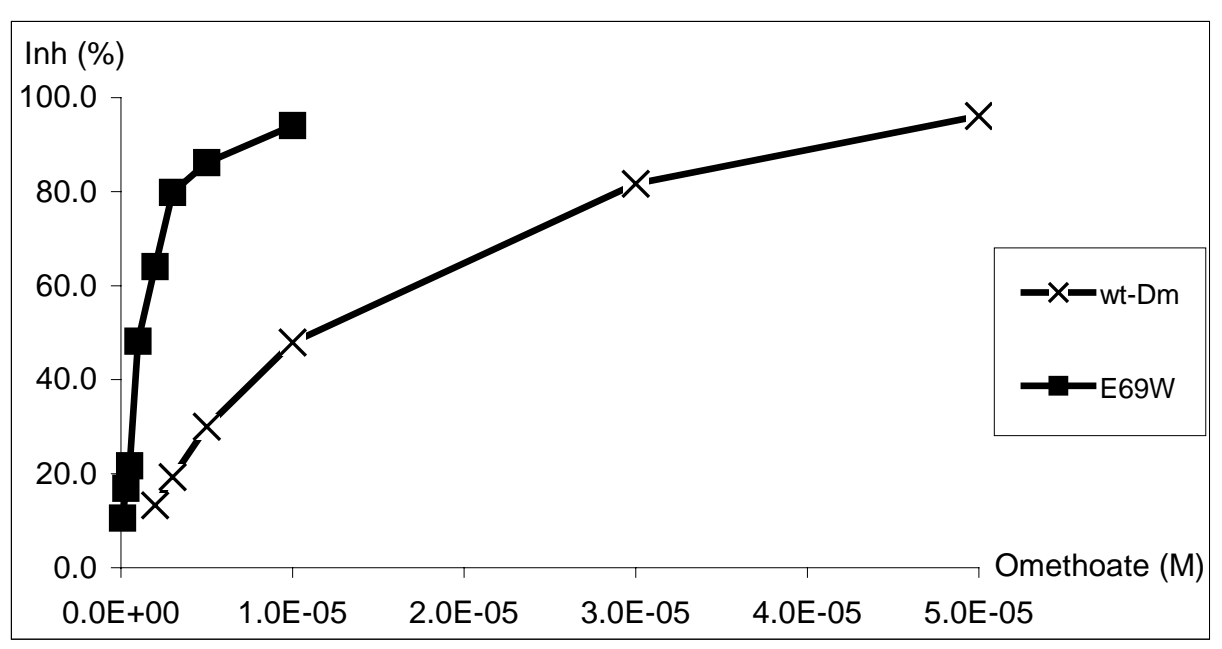

Figure 3. Calibration curves for biosensors based on two different AChE enzymes from Drosophila melanogaster, the wild-type and the genetically modified E69W mutant. Reprinted from [41] with permission from Elsevier.

\subsubsection{Array systems}

A more sophisticated mediator-modified biosensor system is that presented by Law and Higson [66]. A recombinant $\mathrm{AChE}$ was co-entrapped inside an aniline matrix onto a sonicated poly $(o-$ phenylenediamine)-coated CoPC-modified SPE. The sonication process led to the formation of approximately 70000 micro-electrodes $/ \mathrm{cm}^{2}$ at the surface of carbon electrodes. The physical characteristics of the global biosensor were responsible for the favoured hemispherical diffusion of the pesticide to the immobilised enzyme and for the high amplification of the enzyme inhibition. As a result, excellent limits of detection were achieved $\left(10^{-17} \mathrm{M}\right.$ for dichlorvos and $10^{-16} \mathrm{M}$ for parathion methyl and azinphos methyl). Moreover, the system was designed as an array, enabling it to perform multiple measurements at the same time. To improve the biosensor storage stability, a polygalacturonic acid/sucrose stabiliser mixture was added to the electrodes.

Another array system, but in this case designed for simultaneous determination of pesticides and phenols was described by Solná et al. [67]. They designed a four-electrode array system with AChE, $\mathrm{BChE}$, tyrosinase and peroxidase immobilised onto SPEs. The response from both AChE- and BChEmodified electrodes was significantly affected by phenolic compounds, since it was not possible to distinguish if the oxidation current was due to catechol or to thiocholine. Thus, future work must be done to improve the performances of this system.

\subsubsection{Use of novel matrices}

Recent papers report the use of novel matrices to increase the enzyme stability, improve the selectivity and overcome limitations related to the high working potentials. These systems are mainly mediator-free. Sotiropoulou et al. [68] adsorbed AChE on nanostructured conductive carbon, a material with a very low electric resistance. They worked at $+0.1 \mathrm{~V} v s$. $\mathrm{Ag} / \mathrm{AgCl}$, although the optimum working potential was higher $(+0.4 \mathrm{~V})$. Choosing this lower potential, they achieved low limits of detection ( $1 \mathrm{pM}$ for dichlorvos) and prolonged operational lifetime of the sensor. In fact, the 
activated carbon seems to selectively concentrate the pesticide, favouring an enzyme hiperactivity within the nanopores.

Liu et al. [69] presented multiwall carbon nanotubes (CNT) as a novel support for AChE immobilisation by layer-by-layer self-assembling. They compared the performance of carbon nanotube-modified glassy carbon (CNT/GC), glassy carbon, carbon paste and gold electrodes for the determination of thiocholine. Among these, the CNT/GC electrode provided the best sensitivity, reproducibility and limit of detection. Due to its large specific surface area, this material provides an ideal microenvironment for retaining a high enzyme activity. In addition, it favoured the electron transfer. All these characteristics led the authors to use it in the determination of paraoxon [70]. The system, which worked at $+0.15 \mathrm{~V}$ vs. $\mathrm{Ag} / \mathrm{AgCl}$, showed a low background current, minimised the interferences arising from other electroactive compounds and did not present electrode fouling. Moreover, the final system was highly sensitive and stable, being able to detect as low as $4 \times 10^{-13} \mathrm{M}$ paraoxon. Incorporation of an AChE-multiwall-carbon nanotube (MWNT) biosensor into a flow system was recently reported by Kandimalla et al. [71]. The sensor was able to detect concentrations of insecticides ranging from 1.5 to $80 \mu \mathrm{M}$, was reproducible and renewable by reactivation with 2-PAM.

Another work describing the use of a new immobilisation matrix was reported by Shi et al. [72]. In this case, $\mathrm{AChE}$ was entrapped in a $\mathrm{Al}_{2} \mathrm{O}_{3}$ sol-gel. The advantages of this matrix include simple preparation procedure, low cost and increased the enzyme stability, due to its hydrophilicity. Furthermore, a low working potential $(+0.25 \mathrm{~V} v s$. $\mathrm{Ag} / \mathrm{AgCl})$ was applied, due to a direct electron transfer between enzyme and electrode, which improved the selectivity of the system by minimising the interferences.

Another immobilisation matrix is the photocurable PVA-SbQ polymer. This hydrophilic material stabilises the enzyme activity, by physically entrapping the biomolecule. Jeanty et al. [44] integrated a PVA-SbQ/AChE-based biosensor into a continuous flow system. By using the FIA system, the analysis time was greatly improved as compared to the batch mode. In addition, the enzyme was completely regenerated with 2-PAM, a powerful nucleophilic agent, just after the inhibition step. This biosensor system was applied as a tool for the detection of paraoxon and carbaryl for assessing water pollution, and the results were correlated with those obtained by HPLC/UV [73]. The system was later automated (Figure 4) [74]. The new design decreased the cell volume; thus, keeping the same flow rate, they decreased the rinsing time and achieved faster responses. However, this was not favourable for the inhibition kinetics, since the limits of detection were higher than those obtained with the manual system. The authors proposed optimisation of the flow rate as well as the cell volume as a way to decrease the detection limits. Furthermore, they proved the higher inhibition power of the oxon forms of the pesticides and pointed out the advantage of using a prior oxidation step of real samples in order to improve the sensitivity of the system. 


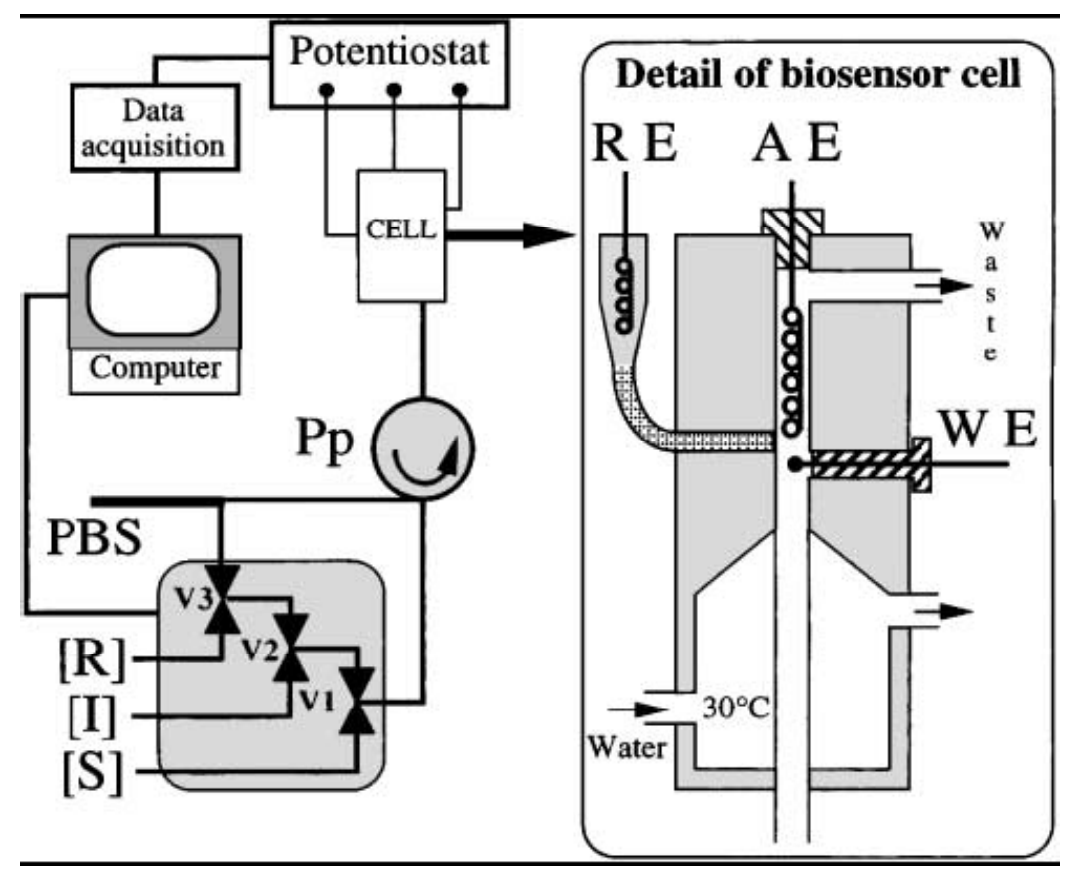

Figure 4. Scheme of the continuous flow system. $\mathrm{V}_{1}, \mathrm{~V}_{2}$ and $\mathrm{V}_{3}$ : electrovalves; [S]: ATChCl solution; [I]: inhibitor solution; [R]: regeneration agent solution; PBS: phosphate buffer solution; Pp: peristaltic pump; RE: reference electrode; AE: auxiliary electrode; WE: working electrode. Reprinted from [74] with permission from Elsevier.

\subsubsection{OPH-based systems}

As mentioned previously, some biosensor systems for pesticide detection are based on the catalytic reaction of the $\mathrm{OPH}$ enzyme. The main advantage of these systems is the direct and single step measurement instead of the multiple steps procedure (inhibition, regeneration, etc.) required when using AChE. Using OPH biosensors, shorter analysis times were obtained, the electrodes could be reused (appropriate for continuous on-line monitoring) and no enzyme substrate was required. Unlike AChE-based biosensors, these sensors are selective only towards organophosphorous compounds that produce $p$-nitrophenol upon hydrolysis. Taking advantage of this simplified procedure, Mulchandani et al. [46] carried out analysis in approximately $2 \mathrm{~min}$, corresponding to a sample throughput of 30 measurements/h. This system provided highly reproducible and stable responses, and excellent storage stability, making it possible to do repeated measurements without regular calibration. Furthermore, broader linear range, higher sensitivity and lower detection limits were achieved compared to potentiometric or optical results. Nevertheless, the high working potential $(+0.9 \mathrm{~V} v \mathrm{vs}$ Ag/AgCl) affected the selectivity of the system. In this direction, the authors mentioned the high selectivity of the OPH-based systems against other widely used pesticides, such as atrazine, sevin, sutan and simazine, and organophosphate insecticides, such as diazinon, which are not hydrolysed by OPH enzyme. Moreover, even though the biosensor system was not selective against pentachlorophenol and phenol, this non-desired current contribution could be subtracted if a blank column (without OPH) is used in parallel. 
A more recent work using OPH enzyme was reported by Wang et al. [47], who immobilised the enzyme onto thin-film gold electrodes instead of inside a reactor. The application of $+0.75 \mathrm{~V} v \mathrm{~s}$. $\mathrm{Ag} / \mathrm{AgCl}$ and the already known problems of surface passivation due to the oxidation of phenolic compounds led the authors to examine the reproducibility of the anodic detection. Results show that the use of low-volume flow injections apparently contributed to a negligible fouling of the electrode surface. The authors are currently translating the system to a lab on a chip using micromachining technology.

\subsubsection{PH-based system}

A higher specific system that uses parathion hydrolase (PH) enzyme was also described [50]. The fact that the parathion is almost insoluble in aqueous solutions suggests the need of an extremely sensitive sensor able to detect low concentrations of this pesticide. As for the OPH-based systems, the detection was based on the oxidation of $p$-nitrophenol, requiring high working potentials. To solve the interference problem, a pulse technique was proposed as detection method. A repetitive potential step from $+0.7 \mathrm{~V}$ to $+0.85 \mathrm{~V}$ vs. $\mathrm{Ag} / \mathrm{AgCl}$ was applied to minimise the effects of other compounds oxidisable below $+0.7 \mathrm{~V}$. The adsorption of the product on the electrode surface was prevented thanks to the constant washing provided by the flow system. As a conclusion, the use of the pulse technique not only eliminates the disturbance of non-specific oxidation reactions, but also increases the sensitivity.

\subsection{Optical and thermal-based systems}

Although most flow-through enzyme sensors for pesticide analysis are electrochemical, some studies reported the use of other detection methods, such as optical and thermal. The scarcity in their utilisation is probably due to the low sensitivity of the spectrophotometric detection, the expensive equipment, the complicated labelling protocols of the fluorescence techniques, and the sophistication of the chemiluminescence and the thermal sensing.

Spectrophotometric detection is simple, easy to implement and cost-effective. However, the sensitivity is not very high and sometimes does not satisfy the screening demands. The simplest strategy is based on the colour change of a $\mathrm{pH}$ indicator as a result of the acetic acid formed during the enzymatic hydrolysis. In this direction, Andres and Narayanaswamy [75] immobilised AChE covalently on isothiocyanate glass beads, mixed them with thymol blue indicator-modified aminopropyl glass beads, and packed them together at the tip of a fibre-optic sensor head integrated into a flow-cell. The LODs for carbofuran and paraoxon were 3.1 and $24.7 \mu \mathrm{g} / \mathrm{L}$, respectively. The main drawback was, however, the rather long response time (16-22 min). In a similar strategy but with chlorophenol red as $\mathrm{pH}$ indicator, Xavier et al. [76] detected propoxur with a LOD of $8 \mu \mathrm{g} / \mathrm{L}$. This biosensor was applied to the analysis of spiked vegetables.

Another spectrophotometric strategy is based on the absorbance measurement of a coloured compound produced by the reaction between thiocholine and Ellman's reagent. Dănet et al. [76] combined a reactor with AChE immobilised on aminated glass pearls with a spectrophotometric detector in a FIA system. The system was applied for the analysis of paraoxon; a working range from 2 
to $80 \mu \mathrm{g} / \mathrm{L}$ and a $\mathrm{LOD}$ of $1.3 \mu \mathrm{g} / \mathrm{L}(\mathrm{RSD}=15 \%)$ were reported. The method only required filtration and $\mathrm{pH}$ adjusting of the water sample. Moreover, the enzyme was reactivated (with 2-PAM) and reused more than 40 times. Although the method is presented as an alarm system, its performance does not fulfil the EU Water Framework Directive requirements for water monitoring. The same strategy, but using magnetic particles, was developed as a comparison method of an automated amperometric FIA system [64]. In this case, the LODs were 3, 3, 10 and $20 \mu \mathrm{g} / \mathrm{L}$ for carbofuran, paraoxon-ethyl, paraoxon-methyl and malaoxon, respectively. Once again, the limitation on sensitivity is obvious. She and Stein [78] lowered the working range for paraoxon from $2-20 \mu \mathrm{g} / \mathrm{L}$ to $0.05-0.5 \mu \mathrm{g} / \mathrm{L}$ just by changing the inhibition time from 3 to $30 \mathrm{~min}$. Consequently, there is a compromise between short analysis times and low sensitivities. Generally, by carefully optimizing the experimental variables, the analytical performance of such biosensors can be easily improved.

The $\mathrm{pH}$ change can also be monitored using a $\mathrm{pH}$-sensitive fluorescence indicator. The inherent high sensitivity of the fluorescence detection should, in principle, overcome the drawbacks of the spectrophotometric detection. In this direction, Navas Díaz and Ramos Peinado [34] immobilised AChE in a sol-gel matrix on a methacrylate plate attached to an optical fiber. They used indoxyl acetate as a substrate, which is enzymatically hydrolysed to a highly fluorescent product. They detected fenitrothion and naled with LODs of 17.8 and $0.36 \mathrm{mg} / \mathrm{L}$. These sensitivities were improved when the enzyme was directly immobilised on the optical fiber (LOD $=0.5$ and $0.12 \mathrm{mg} / \mathrm{L}$ ). Nevertheless, they are not satisfactory enough. A similar strategy was used by Doong and Tsai [79]. Among the different fluorescent indicators, they selected fluorescein isothiocyanate-dextran (FITCdextran) due to the low leaching rate, low toxicity to $\mathrm{AChE}$ and high sensitivity. As low as $152 \mu \mathrm{g} / \mathrm{L}$ paraoxon was detected, which was much lower than the previously reported LODs. Better sensitivities could be achieved by increasing the incubation time, decreasing the flow rate and operating with stopped-flow.

Chemiluminescence is another method that has been used for the detection of the enzymatic inhibition by pesticides [80]. In this case, alkaline phosphatase (ALP) was immobilised together with a polymer on a glass surface connected to a photomultiplier. This enzyme catalyses the dephosphorylation of a macrocyclic compound, releasing light. Detection of paraoxon at a concentration of $1.2 \mu \mathrm{g} / \mathrm{L}$ was achieved, demonstrating the viability of the chemiluminescence-based sensing systems for pesticide detection.

Photothermal spectrometry is based on indirect absorbance measurement by a photothermal effect, arising from the non-radiative relaxation of excited molecules in a sample, which results in defocusing of a laser beam. During the deexcitation, the absorbed energy is converted to heat, which can be measured with a thermal lens. The method was applied to the analysis of paraoxon, diazinon, chlorpyrifos, carbaryl, carbofuran and their mixtures. Compared to the spectrophotometric detection, this technique provided almost 5-times lower LODs and an analysis time of $15 \mathrm{~min}$ [81]. The level of heat produced by the enzymatic reaction can also be monitored with a thermopile sensor. Using chicken liver-esterase, a cheap and highly sensitive enzyme, Zheng et al. [82] detected dichlorvos with a flow injection calorimetric biosensor system. Although they do not report any LOD, the results demonstrate the feasibility of the approach. 


\section{Conclusions}

This paper reviewed the most important biosensor configurations integrated in a flow injection system that have been applied to the detection of organophosphate and carbamate pesticides. Enzymatic systems based on AChE (inhibition-based), $\mathrm{OPH}$ and $\mathrm{PH}$ (direct detection) with electrochemical, optical or thermal detection are discussed in detail. Immunosensors can also be used. Enzyme biosensors are evaluating a total toxicity, while immunosensors are very specific towards a single pesticide for which they are designed. Sensors based on OPH can only detect pesticides that produce p-nitrophenol. Basically, any biosensor capable of flow through measurements can be used as a detector in a FIA system. The work focused primarily on the use of enzyme sensors. The effects of flow parameters (flow rate, incubation time, cell volume), immobilization matrix and use of electronic mediators on the sensitivity and the detection limit are also discussed. As compared to the conventional batch systems, the FIA systems offer the advantages of small sample volumes, continuous substrate injection, continuous enzyme washing for its reactivation, automation and high sample throughput.

In summary, existing flow-based biosensing systems allow detection of pesticides with adequate sensitivity required in public safety and environmental monitoring. However, these devices still have limitations that impede their full exploitation and require further optimisation with respect to the selectivity, stability, functionality in complex matrices, real-time/near real-time detection and in-field monitoring. Recent developments in the field of bioelectronics and materials sciences can considerably improve their analytical performances. In the future, these systems could find use as 'immediate screening devices' capable of rapid identification of samples containing neurotoxic pesticides.

\section{Acknowledgements}

Dr. Campàs acknowledges the European Commission for financial support through the project "Novel technology for controlling wine production and quality (HPRN-CT-2002-00186)". Dr. PrietoSimón acknowledges GTP Technology for financial support.

\section{References}

1. Skládal, P.; Nunes, G.S.; Yamanaka, H.; Ribero, M.L. Detection of carbamate pesticides in vegetable samples using cholinesterase-based biosensors. Electroanalysis 1997, 9, 1083-1087.

2. Pogačnik, L.; Franko, M. Detection of organophosphate and carbamate pesticides in vegetable samples by photothermal biosensor. Biosens. Bioelectron. 2003, 18, 1-9.

3. Donarski, W.J.; Dumas, D.P.; Heitmeyer, D.P.; Lewis, V.E.; Raushel, F.M. Structure-activity relationships in the hydrolysis of substrates by the phosphotriesterase from Pseudomonas diminuta. Biochemistry 1989, 28, 4650-4655.

4. Tuovinen, K.; Kaliste-Korhonen, E.; Raushel, F.M.; Hanninen, O. Phosphotriesterase-A promising candidate for use in detoxification of organophosphates. Fundam. Appl. Toxicol. 1994, 23, 578584.

5. Corrigan, F.M.; Macdonald, S.; Brown, A.; Armstrong, K.; Armstrong, E.M. Neurasthenic fatigue, chemical sensitivity and GABAa receptor toxins. Med. Hypotheses 1994, 43, 195-200. 
6. Ashani, Y.; Rothschild, N.; Segall, Y.; Levanon, D.; Raveh, L. Prophylaxis against organophosphate poisoning by an enzyme hydrolysing organophosphorus compounds in mice. Life Sci. 1991, 49, 367-374.

7. http://ecoport.org/Resources/Refs/Pesticid/Code/PM_Code.htm

8. http://ecoport.org/Resources/Refs/Pesticid/residue.htm

9. http://ec.europa.eu/environment/water/water-framework/index_en.html

10. http://ec.europa.eu/food/plant/protection/index_en.htm

11. http://www.epa.gov/pesticides/

12. http://www.epa.gov/oppfod01/fqpa/

13. http://www.cdc.gov/niosh/topics/pesticides/

14. Aprea, C.; Colosio, C.; Mammone, T.; Minoia, C.; Maroni, M. Biological monitoring of pesticide exposure: a review of analytical methods. J. Chromatogr. B 2002, 769, 191-219.

15. Diehl-Faxon, J.; Ghindilis, A.L.; Atanasov, P.; Wilkins, E. Direct electron transfer based trienzyme electrode for monitoring of organophosphorus pesticides. Sens. Actuat. B 1996, 36, 448457.

16. Di Corcia, A.; Marchetti, M. Multiresidue method for pesticides in drinking water using a graphitized carbon black cartridge extraction and liquid chromatographic analysis. Anal. Chem. 1991, 63, 580-585.

17. Mulchandani, A.; Chen, W.; Mulchandani, P.; Wang, J.; Rogers, K.R. Biosensors for direct determination of organophosphate pesticides. Biosens. Bioelectron. 2001, 16, 225-230.

18. Rekha, K.; Thakur, M. S.; Karanth, N.G. Biosensors for the detection of organo-phosphorous pesticides. Crit. Rev. Biotechnol. 2000, 20, 213-235.

19. Schmid, R.D.; Künnecke, W. Flow injection analysis (FIA) based on enzymes or antibodies applications in the life sciences. J. Biotechnol. 1990, 14, 3-31.

20. Schmidt, H.-L. Biosensors and flow injection analysis in bioprocess control. J. Biotechnol. 1993, 31, v-vi.

21. Gorton, L.; Csöregi, E.; Domínguez, E.; Emnéus, J.; Jönsson-Pettersson, G.; Marko-Varga, G.; Persson, B. Selective detection in flow analysis based on the combination of immobilized enzymes and chemically modified electrodes. Anal. Chim. Acta 1991, 250, 203-248.

22. Tran-Minh, C. Biosensors in flow-injection systems for biomedical analysis, process and environmental monitoring. J. Molec. Recogn. 1996, 9, 658-663.

23. Marco, M.-P.; Gee, S.; Hammock, B.D. Immunochemical techniques for environmental analysis. I. Immunosensors. Trends Anal. Chem. 1995, 14, 341-350.

24. Hock, B.; Dankwardt, A.; Kramer, K.; Marx, A. Immunochemical techniques: Antibody production for pesticide analysis. A review. Anal. Chim. Acta 1995, 311, 393-405.

25. González-Martínez, M.A.; Puchades, R.; Maquieira, A. On-line immunoanalysis for environmental pollutants: from batch assays to automated sensors. Trends Anal. Chem. 1999, 18, 204-218.

26. Mallat, E.; Barzen, C.; Klotz, A.; Brecht, A.; Gauglitz, G.; Barceló, D. River analyzer for chlorotriazines with a direct optical immunosensor. Environ. Sci. Technol. 1999, 33, 965-971.

27. González-Martínez, M.A.; Morais, S.; Puchades, R.; Maquieira, A.; Abad, A.; Montoya, A. Monoclonal antibody-based flow-through immunosensor for analysis of carbaryl. Anal. Chem. 1997, 69, 2812-2818. 
28. Gascón, J.; Oubiña, A.; Ballesteros, B.; Barceló, D.; Camps, F.; Marco, M.-P.; González-Martínez, M.A.; Morais, S.; Puchades, R.; Maquieira, A. Development of a highly sensitive enzyme-linked immunoassay for atrazine. Performance evaluation by flow injection immunoassay. Anal. Chem. 1997, 69, 2812-2818.

29. González-Martínez, M.A.; Penalva, J.; Puchades, R.; Maquieira, A.; Ballesteros, B.; Marco, M.-P.; Barceló, D. An immunosensor for the automatic determination of the antifouling agent Irgarol 1051 in natural waters. Environ. Sci. Technol. 1998, 32, 3442-3447.

30. Bauer, C.G.; Eremenko, A.V.; Ehrentreich-Fŏrster, E.; Bier, F.F.; Makower, A.; Halsall, H.B.; Heineman, W.R.; Scheller, F.W. Zeptomole-detecting biosensor for alkaline phosphatase in an electrochemical immunoassay for 2,4-dichlorophenoxyacetic acid. Anal. Chem. 1996, 68, 24532458.

31. Aldridge W.N. Some properties of specific cholinesterase with particular reference to the mechanism of inhibition by diethyl p-nitrophenyl thiophosphate (E 605) and analogues. Biochem. J. 1950, 46, 441-460.

32. Andres, R.T.; Narayanaswamy, R. Fibre-optic pesticide biosensor based on covalently immobilized acetylcholinesterase and thymol blue. Talanta 1997, 44, 1335-1352.

33. Xavier, M.P.; Vallejo, B.; Marazuela, M.D.; Moreno-Bondi, M.C.; Baldini, F.; Falai, A. Fiber optic monitoring of carbamate pesticides using porous glass with covalently bound chlorophenol red. Biosens. Bioelectron. 2000, 14, 895-905.

34. Navas Díaz, A.; Ramos Peinado, M.C. Sol-gel cholinesterase biosensor for organophosphonus pesticide fluorimetric analysis. Sens. Actuat. B 1997, 38-39, 426-431.

35. Doong, R.-A.; Tsai, H.-C. Immobilization and characterization of sol-gel-encapsulated acetylcholinesterase fiber-optic biosensor. Anal. Chim. Acta 2001, 434, 239-246.

36. Lee, H.-S.; Kim, Y.A.; Cho, Y.A.; Lee, Y.T. Oxidation of organophosphorus pesticides for the sensitive detection by a cholinesterase-based biosensor. Chemosphere 2002, 46, 571-576.

37. Simonian, A.L.; Rainina, E.I.; Wild, J.R. A new approach for discriminative detection fo organophosphate neurotoxins in the presence of other cholinesterase inhibitors. Anal. Lett. 1997, 30, 2453-2468.

38. Evtugyn, G.A.; Ivanov, A.N.; Gogol, E.V.; Marty, J.-L.; Budnikov, H. C. Amperometric flowthrough biosensor for the determination of cholinesterase inhibitors. Anal. Chim. Acta 1999, 385, 13-21.

39. Khayyami, M.; Pérez Pita, M.T.; Peña Garcia, N.; Johansson, G.; Danielsson, B.; Larsson, P.-O. Development of an amperometric biosensor based on acetylcholine esterase covalently bound to a new support material. Talanta 1998, 45, 557-563.

40. Neufeld, T.; Eshkenazi, I.; Cohen, E.; Rishpon, J. A micro flow injection electrochemical biosensor for organophosphorus pesticides. Biosens. Bioelectron. 2000, 15, 323-329.

41. Bucur, B.; Dondoi, M.; Dănet, A.; Marty, J.-L. Insecticide identification using a flow injection analysis system with biosensors based on various cholinesterases. Anal. Chim. Acta 2005, 539, 195-201.

42. La Rosa, C.; Pariente, F.; Hernández, L.; Lorenzo, E. Amperometric flow-through biosensor for the determination of pesticides. Anal. Chim. Acta 1995, 308, 129-136. 
43. Pariente, F.; La Rosa, C.; Galan, F.; Hernández, L.; Lorenzo, E. Enzyme support systems for biosensor applications based on gold-coated nylon meshes. Biosens. Bioelectron. 1996, 11, 11151128.

44. Jeanty, G.; Marty, J.-L. Detection of paraoxon by continuous flow system based enzyme sensor. Biosens. Bioelectron. 1998, 13, 213-218.

45. Rainina, E.I.; Efremenco, E.N.; Varfolomeyev, S.D.; Simonian, A.L.; Wild, J.R. The development of a new biosensor based on recombinant $E$. coli for the direct detection of organophosphorus neurotoxins. Biosens. Bioelectron. 1996, 11, 991-1000.

46. Mulchandani, P.; Chen, W.; Mulchandani, A. Flow injection amperometric enzyme biosensor for direct determination of organophosphate nerve agents. Environ. Sci. Technol. 2001, 35, 25622565 .

47. Wang, J.; Krause, R.; Block, K.; Musameh, M.; Mulchandani, A.; Schöning, M.J. Flow injection amperometric detection of OP nerve agents based on an organophosphorus-hydrolase biosensor detector. Biosens. Bioelectron. 2003, 18, 255-260.

48. Wang, J.; Krause, R.; Block, K.; Musameh, M.; Mulchandani, A.; Mulchandani, P.; Chen, W.; Schöning, M.J. Dual amperometric-potentiometric biosensor detection system for monitoring organophosphorus neurotoxins. Anal. Chim. Acta 2002, 469, 197-203.

49. Schöning, M.J.; Krause, R.; Block, K.; Musahmeh, M.; Mulchandani, A.; Wang, J. A dual amperometric/potentiometric FIA-based biosensor for the distinctive detection of organophosphorus pesticides. Sens. Actuat. B 2003, 95, 291-296

50. Sacks, V.; Eshkenazi, I.; Neufeld, T.; Dosoretz, C.; Rishpon, J. Immobilized parathion hydrolase: An amperometric sensor for parathion. Anal. Chem. 2000, 72, 2055-2058.

51. Trojanowicz, M. Determination of pesticides using electrochemical enzymatic biosensors. Electroanalysis 2002, 14, 1311-1328.

52. Solé. S.; Merkoci, A.; Alegret, S. Determination of toxic substances based on enzyme inhibition. Part II. Electrochemical biosensors for the determination of pesticides using flow systems. Crit. Rev. Anal. Chem. 2003, 33, 127-143.

53. Lee, H.-S.; Kim, Y.-A.; Chung, D.H.; Lee, Y.-T. Determination of carbamate pesticides by a cholinesterase-based flow injection biosensor. Int. J. Food Sci. Technol. 2001, 36, 263-270.

54. Chung, M.-S.; Lee, Y.-T.; Lee, H.-S. Flow injection biosensor for the detection of anticholinesterases. J. Biochem. Mol. Biol. 1998, 31, 296-302.

55. Kumaran, S.; Tran-Minh, C. Determination of organophosphorous and carbamate insecticides by flow injection analysis. Anal. Biochem. 1992, 200, 187-194.

56. Aaron, J.-J.; Tran-Minh, C.; Colliss, J.S.; Smart, N.A. The analyses of fungicides, herbicides and insecticides. Anal. Proc. 1993, 30, 72-77.

57. Ivnitskii, D.M.; Rishpon, J. A potentiometric biosensor for pesticides based on the thiocholine hexacyanoferrate (III) reaction. Biosens. Bioelectron. 1994, 9, 569-576.

58. Nikolelis, D.P.; Simantiraki, M.G.; Siontorou, C.G.; Toth, K. Flow injection analysis of carbofuran in foods using air stable lipid film based acetylcholinesterase biosensor. Anal. Chim. Acta 2005, 537, 169-177. 
59. Suwansa-ard, S.; Kanatharana, P.; Asawatreratanakul, P.; Limsakul, C.; Wongkittisuksa, B.; Thavarungkul, P. Semi disposable reactor biosensors for detecting carbamate pesticides in water. Biosens. Bioelectron. 2005, 21, 445-454.

60. Rodrigues, T.C.; Tubino, M.; Godinho, O.E.S.; de Oliveira Neto, G. An immobilized acetylcholinesterase flow-injection conductimetric system for the determination of paraoxon. Anal. Sci. 1997, 13, 423-427.

61. Botrè, F.; Lorenti, G.; Mazzei, F.; Simonetti, G.; Porcelli, F.; Botrè, C.; Scibona G. Cholinesterase based bioreactor for determination of pesticides. Sens. Actuat. B 1994, 19, 689-693.

62. Pariente, F.; Hernández, L.; Lorenzo, E. 4-Aminophenyl acetate as a substrate for amperometric esterase sensors. Anal. Chim. Acta 1993, 273, 399-407.

63. La Rosa, C.; Pariente, F.; Hernández, L.; Lorenzo, E. Determination of organophosphorus and carbamic pesticides with an acetylcholinesterase amperometric biosensor using 4-aminophenyl acetate as substrate. Anal. Chim. Acta 1995, 295, 273-282.

64. Günther, A.; Bilitewski, U. Characterisation of inhibitors of acetylcholinesterase by an automated amperometric flow-injection system. Anal. Chim. Acta 1995, 300, 117-125.

65. Rippeth, J.J.; Gibson, T.D.; Hart, J.P.; Hartley, I.C.; Nelson, G. Flow-injection detector incorporating a screen-printed disposable amperometric biosensor for monitoring organophosphate pesticides. Analyst 1997, 122, 1425-1429.

66. Law, K.A.; Higson, S.P.J. Sonochemically fabricated acetylcholinesterase micro-electrode arrays within a flow injection analyzer for the determination of organophosphate pesticides. Biosens. Bioelectron. 2005, 20, 1914-1924.

67. Solná, R.; Sapelnikova, S.; Skládal, P.; Winther-Nielsen, M.; Carlsson, C.; Emnéus, J.; Ruzgas, T. Multienzyme electrochemical array sensor for determination of phenols and pesticides. Talanta 2005, 65, 349-357.

68. Sotiropoulou, S.; Chaniotakis, N.A. Lowering the detection limit of the acetylcholinesterase biosensor using a nanoporous carbon matrix. Anal. Chim. Acta 2005, 530, 199-204.

69. Liu, G.; Riechers, S.L.; Mellen, M.C.; Lin, Y. Sensitive electrochemical detection of enzymatically generated thiocholine at carbon nanotube modified glassy carbon electrode. Electrochem. Commun. 2005, 7, 1163-1169.

70. Liu, G.; Lin, Y. Biosensor based on self-assembling acetylcholinesterase on carbon nanotubes for flow injection/amperometric detection of organophosphate pesticides and nerve agents. Anal. Chem. 2006, 78, 835-843.

71. Kandimalla, V. B.; H.X. Ju. Binding of acetylcholinesterase to multiwall carbon nanotube-crosslinked chitosan composite for flow-injection amperometric detection of an organophosphorous insecticide. Chem. Eur. J. 2006, 12, 1074-1080.

72. Shi, M.; Xu, J.; Zhang, S.; Liu, B.; Kong, J. A mediator-free screen-printed amperometric biosensor for screening of organophosphorus pesticides with flow-injection analysis (FIA) system. Talanta 2006, 68, 1089-1095.

73. Marty, J.-L.; Mionetto, N.; Lacorte, S.; Barceló, D. Validation of an enzymatic biosensor with various liquid chromatographic techniques for determining organophosphorus pesticides and carbaryl in freeze-dried waters. Anal. Chim. Acta 1995, 311, 265-271. 
74. Jeanty, G.; Ghommidh, Ch.; Marty, J.-L. Automated detection of chlorpyrifos and its metabolites by a continuous flow system-based enzyme sensor. Anal. Chim. Acta 2001, 436, 119-128.

75. Andres, R.T.; Narayanaswamy, R. Fibre-optic pesticide biosensor based on covalently immobilized acetylcholinesterase and thymol blue. Talanta 1997, 44, 1335-1352.

76. Xavier, M.P.; Vallejo, B.; Marazuela, M.D.; Moreno-Bondi, M.C.; Baldini, F.; Falai, A. Fiber optic monitoring of carbamate pesticides using porous glass with covalently bound chlorophenol red. Biosens. Bioelectron. 2000, 14, 895-905.

77. Dănet, A.F.; Bucur, B.; Cheregi, M.-C.; Badea, M.; Şerban, S. Spectrophotometric determination of organophosphoric insecticides in a FIA system based on AChE inhibition. Anal. Lett. 2003, 36, 59-73.

78. Shi, R.; Stein, K. Flow injection analysis of paraoxon with the use of an immobilized acetylcholinesterase reactor. Anal. Chim. Acta 1996, 324, 21-27.

79. Doong, R.-A.; Tsai, H.-C. Immobilization and characterization of sol-gel-encapsulated acetylcholinesterase fiber-optic biosensor. Anal. Chim. Acta 2001, 434, 239-246.

80. Ayyagari, M.S.; Kamtekar, S.; Pande, R.; Marx, K.A.; Kumar, J.; Tripathy, S.K.; Kaplan, D.L. Biosensors for pesticide detection based on alkaline phosphatase-catalyzed chemiluminescence. Mat. Sci. Eng. C 1995, 2, 191-196.

81. Pogačnik, L.; Franko, M. Determination of organophosphate and carbamate pesticides in spiked samples of tap water and fruit juices by a biosensor with photothermal detection. Biosens. Bioelectron. 1999, 14, 569-578.

82. Zheng, Y.-H.; Hua, T.-C.; Sun, D.-W.; Xiao, J.-J.; Xu, F.; Wang, F.-F. Detection of dichlorvos residue by flow injection calorimetric biosensor based on immobilized chicken liver esterase. $J$. Food Eng. 2006, 74, 24-29.

(C) 2006 by MDPI (http://www.mdpi.org). Reproduction is permitted for noncommercial purposes. 